\title{
Characterization system for resonant micro- and nanocantilevers
}

\section{Sandberg, Rasmus Kousholt; Boisen, Anja; Svendsen, Winnie Edith}

\section{Published in:}

Review of Scientific Instruments

Link to article, DOI:

$10.1063 / 1.2140284$

Publication date:

2005

Document Version

Publisher's PDF, also known as Version of record

Link back to DTU Orbit

Citation (APA):

Sandberg, R. K., Boisen, A., \& Svendsen, W. E. (2005). Characterization system for resonant micro- and nanocantilevers. Review of Scientific Instruments, 76(12), 125101. https://doi.org/10.1063/1.2140284

\section{General rights}

Copyright and moral rights for the publications made accessible in the public portal are retained by the authors and/or other copyright owners and it is a condition of accessing publications that users recognise and abide by the legal requirements associated with these rights.

- Users may download and print one copy of any publication from the public portal for the purpose of private study or research.

- You may not further distribute the material or use it for any profit-making activity or commercial gain

- You may freely distribute the URL identifying the publication in the public portal

If you believe that this document breaches copyright please contact us providing details, and we will remove access to the work immediately and investigate your claim. 


\title{
Characterization system for resonant micro- and nanocantilevers
}

\author{
Rasmus Sandberg, Anja Boisen, and Winnie Svendsen \\ Department of Micro and Nanotechnology, Technical University of Denmark, \\ Ørsteds Plads Building 345 East Kgs. Lyngby, 2800 Denmark
}

(Received 29 July 2005; accepted 24 October 2005; published online 14 December 2005)

\begin{abstract}
We present a system for characterization of the resonant properties of micro- and nanocantilever sensors. The system has been constructed as a vacuum chamber with capabilities for controlling environmental conditions such as pressure, temperature, and chemical constituents. Characterization can be achieved either electrically or using a specialized laser-optical detection system. The system has been used to characterize the resonant properties of $\mathrm{SiO}_{2}$ cantilevers as well as other resonant structures. We present experimental results of a $\mathrm{SiO}_{2}$ resonant cantilever, showing an exceptional accuracy in resonant frequency determination, and demonstrating the importance of resonance characterization in a controlled environment. () 2005 American Institute of Physics.
\end{abstract}

[DOI: $10.1063 / 1.2140284$ ]

\section{INTRODUCTION}

The development and utilization of resonant micro- and nanocantilevers has increased considerably within the past few years because of their low cost and high sensitivity as measurement and detection probes.

When used as a mass detector, the cantilever surface can be coated with a functionalization layer for adsorption of specific chemical compounds. Adsorbed molecules will add to the mass of the cantilever and thereby cause a change in resonant frequency that can be detected.

The principle of operation as well as areas of applications for cantilever sensors are well described in review articles by, e.g., Stemme, ${ }^{1}$ Lavrik, ${ }^{2}$ and Ziegler; ${ }^{3}$ and much work is being done in the field of fabrication and characterization of resonant cantilevers. ${ }^{4-8}$

State-of-the-art nanocantilever mass sensors have been fabricated with reported estimated sensitivities in the attogram and subattogram regime. ${ }^{9,10}$ For characterization of the resonant properties and optimization of such resonant sensors, we have constructed a characterization system, composed around a vacuum chamber, which holds capabilities for controlling ambient conditions, such as pressure, temperature, and gas constituents, while operating the sensors using a set of electrical interconnections. Special equipment has been constructed to facilitate chemical vapor injection. Detection of the resonant motion is achieved either electrically or by means of a laser-optical detection system, which has been constructed in conjunction with the vacuum chamber.

The uniqueness of this characterization system is given in the versatility of its construction, which facilitates easy configuration for characterization of a wide range of resonant sensor components. This is especially useful in research environments where novel components are being developed in an experimental work process.

\section{DESCRIPTION OF SYSTEM COMPONENTS}

The vacuum chamber for the characterization system is constructed in stainless steel as a cylindrical body with an internal diameter of $150 \mathrm{~mm}$ and a height of $200 \mathrm{~mm}$.

Three gas inlets and an inlet for chemical vapor injection are provided as Swagelok 1/4-inch VCR flanges. All other connections to the chamber have been constructed as ISO-KF flanges.

Figure 1 is a schematic diagram of the system setup. The top of the chamber is constructed as a KF DN-160 blank flange with an encased glass plate for visual or video inspection of the samples. This flange also serves as the main opening hatch to the chamber. In addition to this, a KF DN-16 flange for the pump and one KF DN-25 flange for a pressure sensor have been placed in the back of the chamber body. A KF DN-40 flange has been fitted with an electrical feedthrough using a vacuum tight LEMO $^{\circledR}$ model SWH series $5 \mathrm{~S}$ coupler mounted with a 48-pin hermaphroditic multicontact. A couple of LEMO model FFA.5S straight plugs may then be plugged into this coupler providing electrical connections from the sample inside the chamber to any measurement equipment. An additional KF DN-25 flange has been provided for any auxiliary equipment that needs to be connected to the chamber, e.g., a mass spectrometer for analyzing the gas composition in the chamber. Two 45 mounted KF DN-40 flanges are fitted with glass blanks for optical characterization and positioned so their centerlines intersect in the center of the chamber at the position of an inserted cantilever sample.

A Leybold diaphragm pump is used as a backing pump for a Pfeiffer turbo pump. Using the diaphragm pump alone, a medium vacuum level of $30 \mathrm{~Pa}$ can be attained, and the turbo pump can further reduce the pressure to $0.1 \mathrm{~Pa}$.

The inner volume of the chamber and flanges when no equipment has been inserted is approximately $3640 \mathrm{~cm}^{3}$. Measurements show a pressure increase rate of $6.5 \mathrm{mPa} / \mathrm{s}$ 


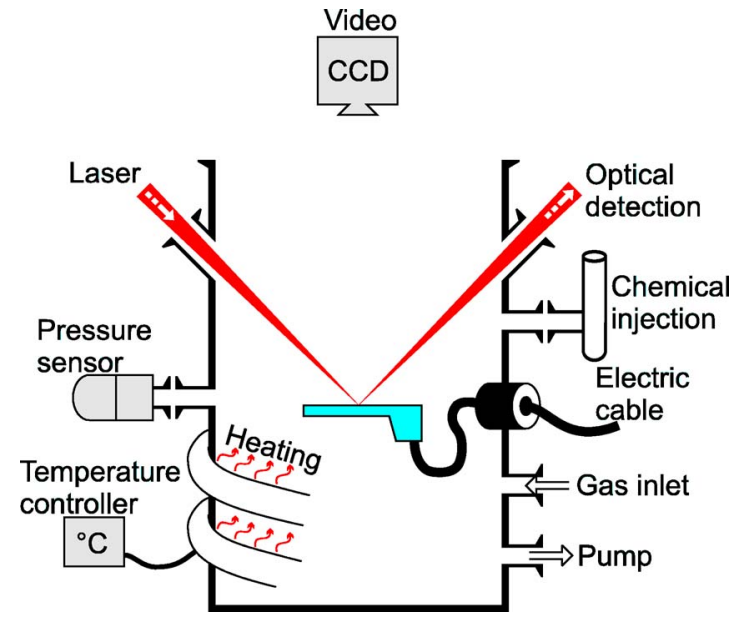

FIG. 1. (Color online) Schematic diagram of the characterization system.

when the chamber is pumped down to vacuum level and the pump valve is closed. This corresponds to a leak rate of about $24 \mathrm{mPa} 1 / \mathrm{s}$.

\section{A. Temperature control}

To control the temperature of the chamber, the exterior surface of the chamber body has been covered with an electrical heating ribbon. At low pressures, heat transfer by convection is almost negligible; and the only way of heating the interior of the chamber is therefore by radiation. For this, the heating ribbon is quite efficient. A delay of approximately $10 \mathrm{~min}$ is observed in the temperature response of the sample temperature to a change in heating power.

The ribbon is connected to a solid-state relay that is driven by a CAL 9400 autotune temperature controller by CAL Controls. The controller is equipped with a RS-232 serial interface, enabling it to be connected to a computer for programmatic control and readout of temperature data.

The temperature sensors connected to the controller are resistance thermometer detectors (RTDs) of thin film platinum (Pt100 type) with an accuracy of $0.06 \%$ at $0{ }^{\circ} \mathrm{C}$, corresponding to $1 / 2$ DIN of the DIN 43760 standard.

\section{B. Gas inlets}

The three VCR gas inlets are placed in the back and at the bottom of the chamber below the sample, in order to have the gas molecules scattered off the sample mount, thus avoiding a direct jet of gas onto the sample.

One inlet is permanently connected to a $\mathrm{N}_{2}$ supply line through a Swagelok bellows valve (model SS-4BKT V51) and an Air Liquide pressure reducer as sketched in Fig. 2.

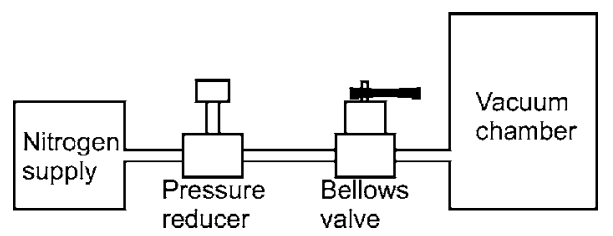

FIG. 2. $\mathrm{N}_{2}$ inlet valve setup (from left to right): The flow of $\mathrm{N}_{2}$ is limited by a pressure reducing valve, followed by a bellows valve (only on/off), immediately before the chamber body.

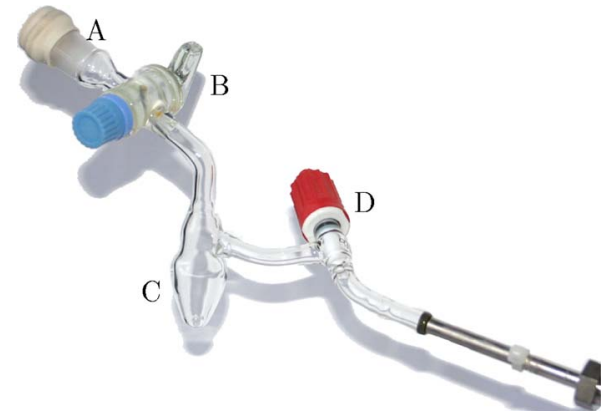

FIG. 3. (Color online) Manifold for injection of chemical vapor. A: latex membrane; B: outer valve; C: chemical manifold; D: vacuum proof Teflon valve.

The bellows valve has only two settings: open and closed, while the pressure reducer allows the flow to be varied continuously. The pressure reducer is not capable of shutting off the flow entirely.

The order of the two valves allows for instantaneous shutoff of the $\mathrm{N}_{2}$ flow into the chamber, but has the disadvantage that the pressure in the pipe section between the two valves will build up when the bellows valve is closed. An initial burst of gas will therefore be let into the chamber when reopening the bellows valve. This burst could be avoided by switching the order of the two valves, which, however, would introduce a different disadvantage, as the $\mathrm{N}_{2}$ pressure between the two valves will be high (supply line pressure) when the bellows valve is open. The high pressure $\mathrm{N}_{2}$ between the valves will therefore continue to flow into the chamber after closing the bellows valve and thus prevent instantaneous shutoff.

The remaining two inlets may in a similar way be connected to any gas supply.

\section{Vapor injection}

A manifold (shown in Fig. 3) has been constructed for injection of chemical vapor into the chamber.

In this device, liquid chemicals may be injected through the membrane (A) and outer valve (B) into the manifold (C), where it will evaporate until the vapor pressure is reached. The vacuum proof Teflon valve (D) may then be opened to let in the amount of vapor needed. The valve allows for very precise control of the chamber pressure up to the chemical vapor pressure. A greater vapor pressure may be obtained by heating up the chamber and manifold.

\section{Electrical connections}

A sample holder has been constructed to facilitate both optical and electrical characterization. The sample holder consists of an $\mathrm{Al}$ base, designed to have a height that ensures that the cantilever sample will be positioned in the center of the chamber, $65 \mathrm{~mm}$ above the chamber floor. This coincides with the intersection of the center axes of the two $45^{\circ}$-angled optical flanges, and is therefore the intended optimum position for the laser focus. The sample holder, shown in Fig. 4, facilitates electrical connections to a sample through a pin grid array (PGA) socket mounted on a printed circuit board (PCB), which is also fitted with two high-intensity white 


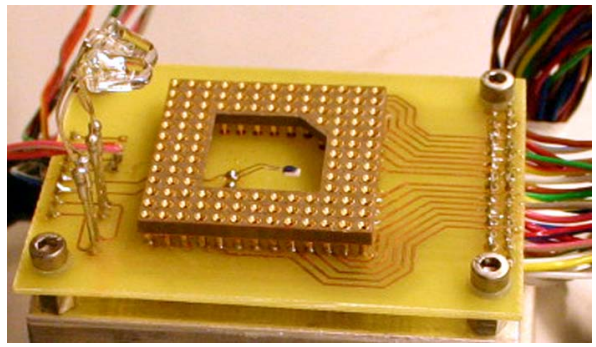

FIG. 4. (Color online) Closeup of the sample holder PCB, showing the PGA socket, one of the thermometer probes, and the two illuminating LEDs.

light emitting diodes for illumination of the mounted sample, and three thermometer probes, of which one is positioned directly underneath the sample and two are connected via cables and may be positioned anywhere in the chamber.

On the outside of the chamber, the conductors are wired to a connection board where external equipment, such as power supplies and measurement instruments may be connected.

In order to be able to actuate passive cantilevers, i.e., cantilevers that are not fabricated with an on-chip actuation mechanism, a chip holder with capabilities of piezoelectric actuation has been constructed (shown in Fig. 5). It fits into the PGA socket and acts as an adapter between the PGA and a flat-flexible-cable (FFC) connector that can be used for 0.4-mm-thick PCBs. These PCBs can then be used as a base onto which a chip containing free hanging cantilevers for out-of-plane oscillation may be fixed by, e.g., epoxy glue. Six electrical connections may be utilized for, e.g., piezoresistive readout. When the PCB is fastened to the FFC connector, it will be in close contact with a piezoelectric ceramic linear transducer element (PZT), which may be driven by a signal generator or similar ac source.

\section{E. Laser-optical detection system}

\section{Introduction}

A laser-optical system was designed and implemented for characterizing the frequency response of resonant cantilevers. The principle is to focus the laser beam onto the cantilever that will reflect the laser at an angle that depends on the deflection (bending) of the cantilever. A position sensitive photodetector (PSD) is then used to measure the deflection of the laser beam and thus the deflection of the can-

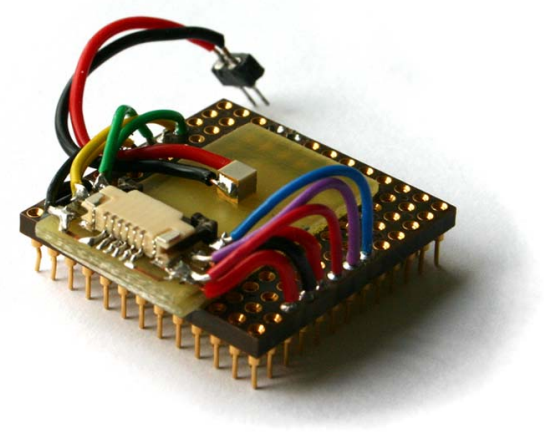

FIG. 5. (Color online) PZT adapter, mounted with a ceramic piezoelectric transducer for actuation of passive cantilever chips.

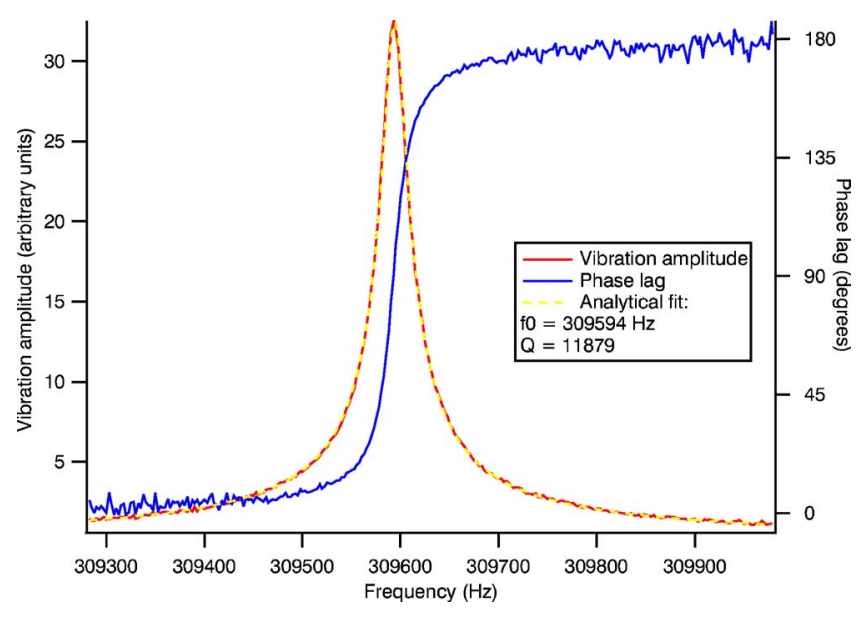

FIG. 6. (Color online) Example of measured data and analytical fit of the vibrational amplitude around the third flexural mode of the $\mathrm{SiO}_{2}$ cantilever in vacuum $(P=10 \mathrm{~Pa})$

tilever. When the cantilever is vibrating, the PSD will generate an ac current with an amplitude proportional to the cantilever's physical deflection amplitude. The principle is similar to that used in atomic force microscopy, developed by Meyer $e t a l .{ }^{11}$

By driving the actuator mechanism of the cantilever with the frequency output of a gain-phase analyzer (GPA), and connecting the PSD output to the GPA input, a frequency sweep can be performed, where the cantilever is put in motion at a range of frequencies and the amplitude and phase response of the cantilever vibration can be measured. If a resonant mode of the cantilever is excited within this frequency range, it will show up on the detected signal as a positive amplitude peak accompanied by a $-\pi$ phase shift, as displayed in Fig. 6.

The optical setup consists of two parts: The focusing part, which guides the laser and focuses in onto the cantilever, and the detection part that collects the reflected light from the cantilever, detects it and amplifies the detector signal. The following two sections will discuss these parts individually.

\section{Laser focusing}

The optical setup is sketched in Fig. 7. The laser beam is redirected on the optical table by the mirrors M1 and M2, expanded and collimated by the lenses L1 and L2 and filtered for noise through the pinhole $\mathrm{PH}$ and the iris I1. The collimated beam is raised vertically and pointed down into the chamber by the mirrors M3 and M4 and focused onto the sample by the lens L3.

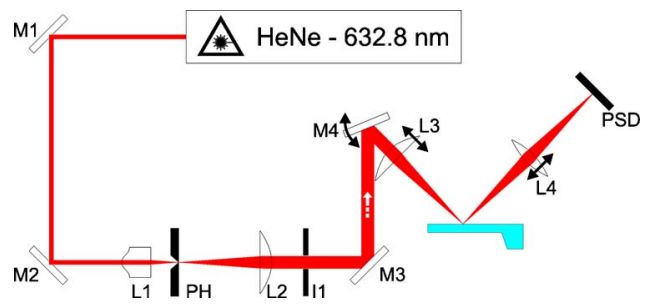

FIG. 7. (Color online) Schematic diagram of the laser-optical detection system. 
The laser is a Spectra-Physics Stabilite model $120 \mathrm{~S}$ helium-neon laser, with an output effect of $7.8 \mathrm{~mW}$ at the wavelength $\lambda=632.8 \mathrm{~nm}$. The waist width is $w_{0}=0.25 \mathrm{~mm}$.

The aspheric lens, L1 and the collimating lens, L2 act as a beam expander. L1 has a focal length of $f_{1}=7.5 \mathrm{~mm}$ and focuses the beam to a waist radius of $1.6 \mu \mathrm{m}$. The position of L1 is adjusted so that this waist coincides with the $10 \mu \mathrm{m}$ diameter pinhole $(\mathrm{PH})$, which filters off most of the nonGaussian spatial noise. The distance from the pinhole to the collimating lens, L2, corresponds to the focal length of L2, $f_{2}=50 \mathrm{~mm}$. The collimated beam has a beam radius of $6.26 \mu \mathrm{m}$ with less than $1 \%$ deviation over $27.5 \mathrm{~m}$. The spatial filtering of the pinhole is not entirely perfect, and the central Gaussian mode of the collimated beam is surrounded by a number of fringes. These are removed by the adjustable iris, I1.

The planoconvex focusing lens, L3, has a focal length $f_{3}=200 \mathrm{~mm}$ and focuses the Gaussian beam onto the sample. The lens is mounted on a micrometer precision translation stage with $30 \mathrm{~mm}$ movement parallel to the beam direction. With this translation stage and the two adjuster screws of the mirror, M4, the focus point of the beam can be adjusted in all three dimensions with micrometer accuracy.

\section{Detection and amplification}

The laser beam, after being focused onto the sample, is reflected up and out through the second angled flange in the chamber wall. Here it is collected by the lens, L4, which focuses the light onto the PSD. The photocurrent generated by the detector is then converted to a voltage, amplified, and measured by, e.g., a gain-phase analyzer.

The detector in the setup is a one-dimensional analog PSD from SiTek, model 1L20_CP3, with an active area of $3 \times 20 \mathrm{~mm}$, and a responsivity of $0.63 \mathrm{~mA} / \mathrm{mW}$. It has two current outputs, and the generated photocurrent from absorbed light is distributed between the outputs with almost linear dependence on the position of the light. Because of the large active area, the theoretically optimal position of the detector is at the position where the deflection of the center of the beam is maximal (within the limit of the $20 \mathrm{~mm}$ height of the detector). The lens, L4, therefore serves no purpose in the ideal case. In praxis, the light reflected from a cantilever is much more diffuse than a Gaussian beam, and the lens is necessary for collecting the diffuse reflections that would otherwise not reach the detector. A PSD position of a few centimeters after the lens has been observed to give optimal results, but the exact position is not critical.

Under the assumption that the laser spot reaching the sample has a Gaussian profile, and that the sample has a perfectly planar, reflective surface that is at least as large as the dimension of the laser spot, the reflected beam can be treated analytically, to evaluate the functionality and estimate the sensitivity of the detection.

A theoretical calculation of the signal currents from the PSD when exposed to a Gaussian laser spot can be done by integrating the Gaussian intensity profile at the PSD position, $I(x, y)$, folded with the position-dependent current response, over the active area of the PSD. This yields a current-signal amplitude of

$$
i_{1}-i_{2}=9.15 \mu \mathrm{A} / \mathrm{mW} / \mathrm{mrad},
$$

which should be multiplied by the absorbed laser power and the deflection angle of the cantilever sample. It is assumed that the two current-outputs are identical $\left(i_{1}-i_{2}=0\right)$ for zero deflection.

The noise current of the PSD is specified to $0.5 \mathrm{pA} / \sqrt{\mathrm{Hz}}$ for each output channel, which gives a rms noise for the current difference of $0.71 \mathrm{pA} / \sqrt{\mathrm{Hz}}$. The theoretical detection limit (signal-to-noise ratio of 1 ) is therefore

$$
\Delta \theta_{\min }=78 \mathrm{prad} \mathrm{mW} / \sqrt{\mathrm{Hz}} \text {. }
$$

With a typical detection bandwidth of $100 \mathrm{~Hz}$ and a reflected laser power of $0.1 \mathrm{~mW}$, the minimum detectable deflection amplitude is then ideally $\Delta \theta_{\min } \approx 8 \mathrm{nrad}$.

When the noise generated in the amplification of the current signal is taken into account, the detection limit of the entire electrical system is slightly reduced and can be evaluated to $\Delta \theta_{\min } \approx 10 \mathrm{nrad}$.

\section{VERIFICATION: RESONANT PROPERTIES OF $\mathrm{SiO}_{2}$ CANTILEVER}

The system has successfully been utilized to characterize a variety of resonant components, including the state-of-theart cantilever mass sensor with integrated complimentary metal-oxide semiconductor (CMOS) actuation and detection that was developed in the EU project "Nanomass" (Refs. 9 and 12), in-plane vibrating cantilever structures, and monolithic silicon dioxide cantilevers ${ }^{13}$

For optimization of resonant cantilever sensors, the two most important parameters to control are the resonant frequency and the $Q$ factor. The actual sensitivity of the sensor is closely related to the accuracy with which the resonant frequency can be measured, which in turn depends on the measurement system as well as the $Q$ factor of the resonant mode. Measurements done on a $\mathrm{SiO}_{2}$ cantilever are presented here to demonstrate the accuracy of the characterization system.

Figure 6 displays the measured data of the third resonant mode of a $\mathrm{SiO}_{2}$ cantilever. The cantilever is rectangular, with dimensions $h \times w \times \ell=0.85 \times 10 \times 211 \mu \mathrm{m}$. It has been actuated using the piezoceramic actuator (PZT) in physical contact with the chip, and the cantilever vibration amplitude was measured using the optical detection system and a gainphase analyzer (GPA). The PZT was driven directly by the frequency reference output of the GPA. Resonant frequency and $Q$ factor can be determined by fitting the resonance peak to the analytical expression for a forced, damped harmonic oscillator as described in, e.g., Ref. 14 and for the vibration amplitude, the measured data points and the fitted function are seen to coincide almost completely. The resonant frequency for this particular mode was determined as $f_{0}$ $=309593.88 \mathrm{~Hz}$ with an accuracy of $0.05 \mathrm{~Hz}$.

The first five flexural modes of the cantilever were measured for a range of pressures. The characterization of higher order modes is interesting because of the increased sensitivity over the fundamental mode. Observing the pressure dependency is important because of the great influence of dissipative and molecular damping on the $Q$ factor of the 


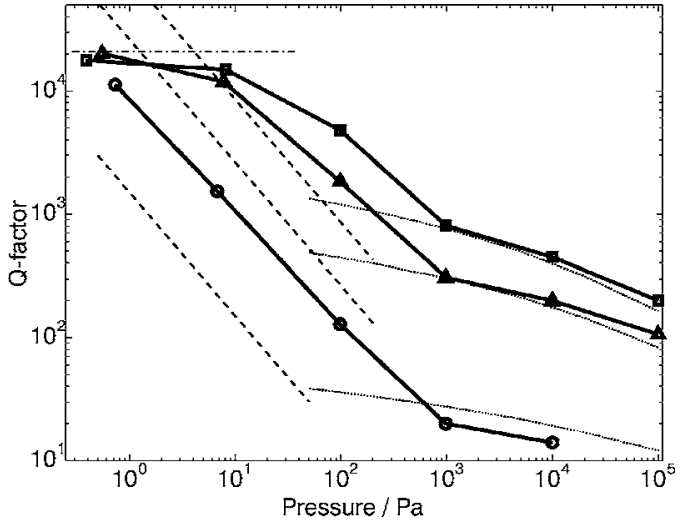

FIG. 8. Measured and modeled $Q$ factors for flexural mode 1 (circles), 3 (triangles), and 5 (squares) of the $\mathrm{SiO}_{2}$ cantilever as a function of Ar pressure. The dissipative damping model is represented by the dotted curve; the dashed curve is the molecular damping; and the dash-dotted line is the upper limit of internal damping.

cantilever. As most cantilever sensor applications require operation at atmospheric pressure, great effort has been put into describing the effect of pressure on the resonant properties of cantilevers-both experimentally ${ }^{15-19}$ and theoretically. ${ }^{20-23}$ The problem remains nontrivial though, and experimental observations are therefore of great importance for characterizing cantilever sensors.

The resonant frequencies of the measured modes in vacuum deviate less than $0.5 \%$ from analytical theory; ${ }^{13}$ a deviation that can be fully accounted for by the uncertainties of the dimensions and material properties.

The measured $Q$ factors are displayed in Fig. 8 as a function of pressure (here in pure argon atmosphere). The $Q$ factors are determined by the dominant source of damping, which depends on the pressure and molecular weight of the gas. In high vacuum, the internal friction of the cantilever and vibrational coupling to the chip substrate dominates, setting an absolute maximum for the $Q$ factor. This limit has been empirically observed for our cantilevers as $Q \approx 20000$ (dash-dotted line). In the pressure regime, where the mean free path of the gas molecules is longer than the cantilever width, molecular damping dominates, resulting in a $Q$ factor that is inversely proportional to the pressure (dashed curves). At pressures above this molecular regime, the gas must be treated as a viscous medium, where the $Q$ factor is limited by viscous damping (dotted curves).

The modeled curves are based on the analytical expression for the $Q$ factor due to molecular damping, provided by Blom et al. in Ref. 20 and the expression for viscous damping that has been derived by Sader et al. in Ref. 23. We see that the viscous model is in very good agreement with the observed $Q$ factors for pressures above $\approx 1 \mathrm{kPa}$, while it overestimates the damping at lower pressures. The molecular model however, apparently overestimates the damping at all pressures, yielding $Q$ factors that are almost an order of magnitude lower than the observed, although the general pressure dependence (the slope of the curve) is in good agreement for pressures below $\approx 1 \mathrm{kPa}$.

The difficulties in theoretical determination of the $Q$ factors of resonant cantilever sensors demonstrate the necessity of experimental characterization of these devices in a controlled environment. The presented characterization system proves valuable for this purpose, and provides a platform for highly accurate measurements of the resonant properties of cantilever and other resonant sensors.

\section{CONCLUSION}

A versatile characterization system for resonant microand nanosensors has been constructed, and its functionality has been demonstrated and utilized to characterize the resonant properties of various sample sensors.

Sensor actuation is done via an electrical interface, and sensor vibration detection may be done either electrically or by means of a laser-optical detection system.

The optical detection system has been proved functional for frequencies in excess of $2 \mathrm{MHz}$, and has been used successfully on out-of-plane as well as in-plane vibrating cantilever sensors, doubly clamped bridge-structure sensors, and monolithic $\mathrm{SiO}_{2}$ cantilevers with low optical reflectivity. ${ }^{13}$

The system is easily configured for different measurement and characterization purposes, and is thus very valuable in an experimental micro- and nanomechanical research institution.

We have presented experimental results that demonstrate an accuracy of the optical detection system of $0.05 \mathrm{~Hz}$ in determining resonant frequency; and by comparing theoretical models with experimental data for the pressure dependence of $Q$ factors, we have demonstrated the importance of experimental data for characterization and optimization of resonant cantilever sensors.

\section{ACKNOWLEDGMENTS}

The authors wish to thank Kristian Mølhave (Department of Micro and Nanotechnology, Technical University of Denmark) for assistance with chip design and fabrication. This work was funded by the EU IST project NANOMASS II (IST-2001-33068), and by the Technical University of Denmark.

${ }^{1}$ G. Stemme, J. Micromech. Microeng. 1, 113 (1991).

${ }^{2}$ N. V. Lavrik, M. J. Sepaniak, and P. G. Datskos, Rev. Sci. Instrum. 75, 2229 (2004).

${ }^{3}$ C. Ziegler, Anal. Chem. 379, 946 (2004).

${ }^{4}$ T. Thundat, G. Y. Chen, R. J. Warmack, D. P. Allison, and E. A. Wachter, Anal. Chem. 67, 519 (1995).

${ }^{5}$ H. Kawakatsu, S. Kawai, D. Saya, M. Nagashio, D. Kobayashi, H. Toshiyoshi, and H. Fujita, Rev. Sci. Instrum. 73, 2317 (2002).

${ }^{6}$ N. V. Lavrik and P. G. Datskos, Appl. Phys. Lett. 82, 2697 (2003).

${ }^{7}$ U. Gysin, S. Rast, P. Ruff, E. Meyer, D. W. Lee, P. Vettiger, and C. Gerber, Phys. Rev. B 69, 045403 (2004).

${ }^{8}$ J. Tamayo, J. Appl. Phys. 97, 044903 (2005).

${ }^{9}$ E. Forsén, G. Abadal, S. Ghatnekar-Nilsson, J. Teva, J. Verd, R. Sandberg, W. Svendsen, F. Peréz-Murano, J. Esteve, E. Figueras, F. Campabadal, L. Montelius, N. Barniol, and A. Boisen, Appl. Phys. Lett. 87, 043507 (2005).

${ }^{10}$ B. Ilic, H. G. Craighead, S. Krylov, W. Senaratne, C. Ober, and P. Neuzil, J. Appl. Phys. 95, 2694 (2004).

${ }^{11}$ G. Meyer and N. M. Amer, Appl. Phys. Lett. 53, 1045 (1988).

${ }^{12}$ European Union IST project NANOMASS II (IST-2001-33068), http:// einstein.uab.es/_c_nanomass

${ }^{13}$ R. Sandberg, W. Svendsen, K. Mølhave, and A. Boisen, J. Micromech. Microeng. 15, 1454 (2005).

${ }^{14}$ G. Y. Chen, R. J. Warmack, T. Thundat, D. P. Allison, and A. Huang, Rev. 
Sci. Instrum. 65, 2532 (1994).

${ }^{15}$ C. Bergaud, L. Nicu, and A. Martinez, Jpn. J. Appl. Phys., Part 1 38, 6521 (1999).

${ }^{16}$ J. Mertens, E. Finot, T. Thundat, A. Fabre, M.-H. Nadal, V. Eyraud, and E. Bourillot, Ultramicroscopy 97, 119 (2003).

${ }^{17}$ K. Yum, Z. Wang, A. P. Suryavanshi, and M.-F. Yu, J. Appl. Phys. 96, 3933 (2004)

${ }^{18}$ J. W. M. Chon, P. Mulvaney, and J. E. Sader, J. Appl. Phys. 87, 3978 (2000).
${ }^{19}$ R. Sandberg, K. Mølhave, A. Boisen, and W. Svendsen, J. Micromech. Microeng. 15, 2249 (2005).

${ }^{20}$ F. R. Blom, S. Bouwstra, M. Elwenspoek, and J. H. J. Fluitman, J. Vac. Sci. Technol. B 10, 19 (1992).

${ }^{21}$ Y.-H. Cho, A. P. Pisano, and R. T. Howe, J. Microelectromech. Syst. 3, 81 (1994).

${ }^{22}$ G. Lévêque, P. Girard, S. Belaidi, and G. Cohen Solal, Rev. Sci. Instrum. 68, 4137 (1997).

${ }^{23}$ J. E. Sader, J. Appl. Phys. 84, 64 (1998) 